\title{
Many Tabs Make a Light Board
}

\author{
Nirmal J. Patel \\ GVU Center, School of Interactive Computing \\ Georgia Institute of Technology \\ Atlanta, GA, USA \\ nirmal@gatech.edu
}

\begin{abstract}
My research examines challenges inherent in the design of mobile groupware systems. For my thesis work I am designing interfaces and interaction techniques that can be used to augment face-to-face communication within groups of collocated mobile users. In my initial research, which explored collocated mobile photo capture and sharing, I uncovered three fundamental challenges to designing mobile groupware. In this paper I discuss these challenges as well as my proposed research agenda to addresses the challenges.

Categories and Subject Descriptors: H.5.2 [Information Systems and Presentation]: User Interfaces

General Terms: Design, Group, Social

Keywords: mobile, collocated, groupware, design

\section{INTRODUCTION}

In Weiser's seminal vision of the future of computing, he described tabs, pads and boards: inch, foot, and yard scale devices. Boards are a platform which knowledge workers could use to engage with information as a group while tabs were devices similar in form factor to today's mobile phone [4]. Large-scale, interactive computing is common in academic research laboratories but is rare for the average consumer, while, the number of commercially available "tabs" has grown exponentially. I believe that the computing power, connectivity, and ubiquity of mobile phones makes them an ideal platform for creating groupware applications that can enhance face-to-face interaction within collocated groups of mobile users.
\end{abstract}

\section{THE CHALLENGES}

In my work to date, I have uncovered three fundamental design challenges in the creation of groupware for mobile devices [3]. The first challenge is the problem of awareness of interactions within the groupware. For example, in table-top applications it is easy for one user to determine the context of another's interactions with the groupware through consequential communication. However, the mobile form factor makes it difficult to peripherally monitor a group member's action, thus requiring more explicit interaction amongst group member.

The second challenge is to provide users with a clear method for having a discussion around common artifacts within the system. I believe, this challenge, is primarily a function

Copyright is held by the author/owner(s). MobileHCI 2010 September 7-10, 2010, Lisboa, Portugal. ACM 978-1-60558-835-3/10/09. of how the form factor of mobile phones encourage, and in some cases require, the user to orient the device towards themselves, thus making it difficult for other users to interact or even observe the phone. Research in this area has focused on implementation of floor control policies [1].

Third, relates to the fact that verbal communication occurs outside of the system. Mobile, collocated groupware will primarily augment face-to-face conversation. Designers can approach this challenge with a range of methods, from remaining ignorant of the conversation to attempting to listen-in and have the system react as a first-class participant in the group experience. My work has shown that with sufficient cues in design, groupware can provide users with a way to resolve diectic references to artifacts within the system [3].

\section{FUTURE WORK}

My research has focused on creating systems which enable mobile, collocated group interactions and evaluating them in real world field studies [3]. A system which I am currently developing involves interaction of users with large scale documents, such as maps, a common application for large-scale displays. This project I hope will stress all three of my highlighted challenges. Prior work has looked at mobile map use; however, most projects have been concerned with multi-user single-device applications [2].

I am also planning on performing a series of controlled experiments to better understand issues presented by the third challenge, specifically the ability of groupware to interject itself into a conversation amongst users. At one extreme, I will explore techniques which do not require understanding of external communication but use interruptive actions, such as vibrating, beeping or flashing of the device screen. These experiments will focus on the ability of users to successfully multiplex the groupware into conversations. At the other extreme, I will look at techniques which incorporate conversation amongst users into the groupware interaction.

\section{References}

[1] A. Kun, M. Leonard, and G. Marsden. Co-present photo sharing on mobile devices. In MobileHCI '0\%. ACM, 2007.

[2] A. Morrison, A. Oulasvirta, P. Peltonen, S. Lemmela, G. Jacucci, G. Reitmayr, J. Näsänen, and A. Juustila. Like bees around the hive. In CHI '09. ACM, 2009.

[3] N. Patel, J. Clawson, A. Voida, and K. Lyons. Mobiphos: A study of user engagement with a mobile collocatedsynchronous photo sharing application. IJHCS, 67(12), 2009.

[4] M. Weiser. The computer for the 21st century. Scientific American, 272(3), 1995. 